\title{
Abstract Voronoi diagrams
}

\author{
Rolf Klein*
}

Universität Bonn, Institut für Informatik I

\begin{abstract}
Abstract Voronoi diagrams are a unifying framework that covers many types of concrete Voronoi diagrams. This talk reports on the state of the art, including recent progress.
\end{abstract}

\section{Introduction}

Concrete Voronoi diagrams [1] are mostly defined in terms of sites and distance, and both concepts can vary greatly. Abstract Voronoi diagrams [6] are built on what most concrete diagrams have in common: a system of simple bisecting curves $J(p, q)=J(q, p)$, where $p, q$ are just indices from a set $S$ of $n$ elements. Each curve $J(p, q)$ divides the plane into two domains, $D(p, q)$ and $D(q, p)$. The abstract Voronoi region of $p$ with respect to $S$ is defined by

$$
\operatorname{VR}(p, S):=\bigcap_{q \in S \backslash\{p\}} D(p, q)
$$

and the abstract Voronoi diagram of $S$ is just the plane minus all Voronoi regions.

An interesting question is what properties to require of the curves $J(p, q)$. They should be as weak as possible for generality, but strong enough to ensure that useful "Voronoi" structures result from the above definitions. It turns out [7] that the following are sufficient.

(A1) Each curve $J(p, q)$, where $p \neq q$, is unbounded. After stereographic projection to the sphere, it can be completed to a closed Jordan curve through the north pole.

For any three indices $p, q, r$ in $S$, and $S^{\prime}:=$ $\{p, q, r\}$,

(A2) each Voronoi region $\operatorname{VR}\left(p, S^{\prime}\right)$ is path-wise connected,

(A3) each point of the plane belongs to the closure of a Voronoi region $\operatorname{VR}\left(p, S^{\prime}\right)$.

*Email: rolf.klein@uni-bonn.de. This work was supported by the European Science Foundation (ESF) in the EUROCORES collaborative research project EuroGIGA/VORONOI.
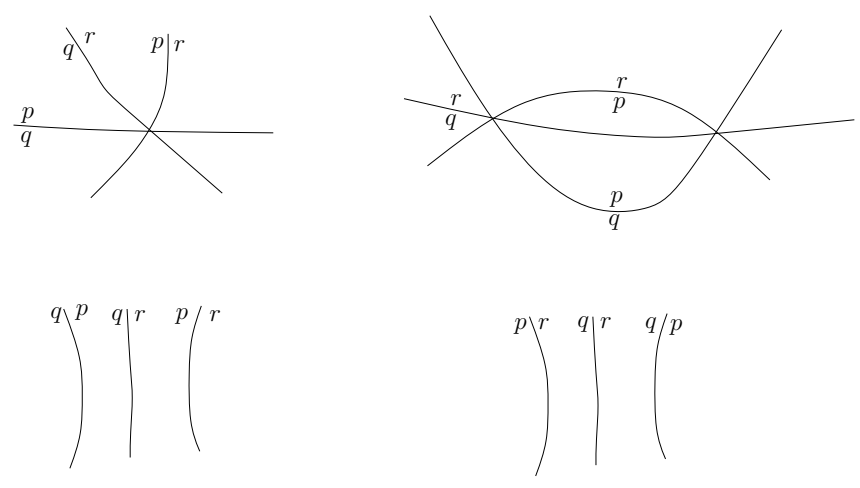

Figure 1: Admissible curve systems

Informally, if the bisecting curves are unbounded and behave decently, and if any triplet $J(p, q), J(p, r), J(q, r)$ is situated as shown in Figure 1 , the AVD theory applies.

\section{Results}

This means that structural results and efficient algorithms become available without further effort [7].

Theorem $1 V(S)$ is a planar graph of complexity $O(n)$. It can be constructed in an expected number of $O(n \log n)$ many steps.

If we replace Axiom A2 by the more general requirement

(A2') Each Voronoi region $\operatorname{VR}\left(p, S^{\prime}\right)$ has at most $s$ connected components

(and assume that any two curves intersect only finitely often), the above result can be generalized as follows [3].

Theorem 2 Abstract Voronoi diagrams with disconnected regions can be computed in an expected number of

$$
O\left(s^{2} n \sum_{j=3}^{n} \frac{m_{j}}{j}\right)
$$

steps, where $m_{j}$ denotes the average number of faces per region in all AVDs of $j$ sites from $S$. 
One can extend the definition of abstract Voronoi diagrams to orders $k>1$ by defining

$$
\operatorname{VR}^{k}(P, S):=\bigcap_{p \in P,} D(p, q) .
$$

For order $k=n-1$, the resulting AVDs are trees [9] of linear size. In the general case the following complexity result holds. Here we assume that all curves are in general position, and that the standard Voronoiregions are non-empty.

Theorem 3 The abstract order-k Voronoi diagram $V^{k}(S)$ has at most $2 k(n-k)$ many faces. This bound can be achieved.

\section{Conclusion}

Open is the case of closed bisecting curves.

\section{References}

[1] F. Aurenhammer, R. Klein, and D.-T. Lee, Voronoi Diagrams and Delaunay Triangulations, World Scientific Publishing Company, to appear August 2013.

[2] C. Bohler, P. Cheilaris, R. Klein, C.H. Liu, E. Papadopoulou, and M. Zavershynskyi, On the complexity of higher order abstract Voronoi diagrams, to be presented at ICALP' 13.

[3] C. Bohler and R. Klein, Abstract Voronoi diagrams with disconnected regions, Bonn 2013, manuscript.

[4] C. Bohler and R. Klein, Point sites with individual distance functions, Bonn 2012, manuscript.

[5] A. G. Corbalan, M. Mazon, and T. Recio, Geometry of bisectors for strictly convex distance functions, International Journal of Computational Geometry and Applications 6(1) (1996), 45-58.

[6] R. Klein, Concrete and abstract Voronoi diagrams, Lecture Notes in Computer Science, 400, SpringerVerlag, 1987.

[7] R. Klein, E. Langetepe, and Z. Nilforoushan, Abstract Voronoi diagrams revisited, Computational Geometry: Theory and Applications 42(9) (2009), 885-902.

[8] R. Klein, K. Mehlhorn, and S. Meiser, Randomized incremental construction of abstract Voronoi diagrams, Computational Geometry: Theory and Applications 3 (1993), 157-184.

[9] K. Mehlhorn, S. Meiser, and R. Rasch, Furthest site abstract Voronoi diagrams, International Journal of Computational Geometry and Applications 11(6) (2001), 583-616. 